International Mathematical Forum, Vol. 8, 2013, no. 20, 973 - 981 HIKARI Ltd, www.m-hikari.com

\title{
Weak Connes Amenability of the Even Duals of a Banach Algebra
}

\author{
Mina Ettefagh \\ Department of Mathematics \\ Tabriz Branch \\ Islamic Azad University \\ Tabriz, Iran \\ minaettefagh@gmail.com \\ Sina Etemad \\ Department of Mathematics \\ Tabriz Branch \\ Islamic Azad University \\ Tabriz, Iran \\ sina.etemad@gmail.com
}

Copyright (c) 2013 Mina Ettefagh and Sina Etemad. This is an open access article distributed under the Creative Commons Attribution License, which permits unrestricted use, distribution, and reproduction in any medium, provided the original work is properly cited.

\begin{abstract}
Let $\mathcal{A}$ be a Banach algebra. In this paper, we introduce the concept of weak Connes amenability of $\mathcal{A}$. Next, by some conditions, we show that how weak amenability of $\mathcal{A}^{(2 n)}$ implies weak Connes amenability of $\mathcal{A}^{(2 n+2)}$. Finally, we relate this notion to the factorization property of odd duals of $\mathcal{A}$.
\end{abstract}

Mathematics Subject Classification: 46H20, 46H25

Keywords: Banach algebra, Arens Regularity, Weak Amenability, Connes Amenability, factorization 


\section{Introduction and Preliminaries}

An Amenable Banach algebra was first introduced by B.E. Johnson in [3]. This notion also appeared in the work of A.Ya. Helemskii [2] which was published in the same year. Then, different generalizations were developed from this structure by other mathematicians. For instance, Dales, Ghahramani and Gronbaek started the concept of $n$-weak amenability of Banach algebras in [5]. Other notion from this disscusion, studied by V. Runde in [8], is Connes amenability of dual Banach algebras.

Let $\mathcal{A}$ be a Banach algebra and $\mathcal{A}^{*}, \mathcal{A}^{* *}, \ldots$ and $\mathcal{A}^{(n)}$ are the first, second, $\ldots$ and $n$-th dual of $\mathcal{A}$, respectively. For $a \in \mathcal{A}$ and $a^{\prime} \in \mathcal{A}^{*}$, we denote by $a^{\prime} a$ and $a a^{\prime}$ respectively, the functionals on $\mathcal{A}^{*}$ defined by $\left\langle a^{\prime} a, b\right\rangle=\left\langle a^{\prime}, a b\right\rangle=$ $a^{\prime}(a b)$ and $\left\langle a a^{\prime}, b\right\rangle=\left\langle a^{\prime}, b a\right\rangle=a^{\prime}(b a)$ for all $b \in \mathcal{A}$.

R. Arens [4] defined two products on the second dual of Banach algebra $\mathcal{A}$ and represented them with symbols $\square$ and $\diamond$. These products are defined in three steps:

$$
\begin{aligned}
\left\langle a^{\prime} a, b\right\rangle & =\left\langle a^{\prime}, a b\right\rangle, \\
\left\langle a^{\prime \prime} a^{\prime}, a\right\rangle & =\left\langle a^{\prime \prime}, a^{\prime} a\right\rangle, \\
\left\langle a^{\prime \prime} \square b^{\prime \prime}, a^{\prime}\right\rangle & =\left\langle a^{\prime \prime}, b^{\prime \prime} a^{\prime}\right\rangle,
\end{aligned}
$$

where $a, b \in \mathcal{A}, a^{\prime} \in \mathcal{A}^{*}$ and $a^{\prime \prime}, b^{\prime \prime} \in \mathcal{A}^{* *}$. In this case, $a^{\prime \prime} \square b^{\prime \prime}$ is called the first Arens product of $a^{\prime \prime}$ and $b^{\prime \prime}$. Similarly

$$
\begin{aligned}
\left\langle a a^{\prime}, b\right\rangle & =\left\langle a^{\prime}, b a\right\rangle, \\
\left\langle a^{\prime} a^{\prime \prime}, a\right\rangle & =\left\langle a^{\prime \prime}, a a^{\prime}\right\rangle, \\
\left\langle a^{\prime \prime} \diamond b^{\prime \prime}, a^{\prime}\right\rangle & =\left\langle b^{\prime \prime}, a^{\prime} a^{\prime \prime}\right\rangle,
\end{aligned}
$$

for all $a, b \in \mathcal{A}, a^{\prime} \in \mathcal{A}^{*}$ and $a^{\prime \prime}, b^{\prime \prime} \in \mathcal{A}^{* *}$. Then $a^{\prime \prime} \diamond b^{\prime \prime}$ is called the second Arens product of $a^{\prime \prime}$ and $b^{\prime \prime}$. The Banach algebra $\mathcal{A}$ is said an Arens regular if and only if $a^{\prime \prime} \square b^{\prime \prime}=a^{\prime \prime} \diamond b^{\prime \prime}$ for every $a^{\prime \prime}, b^{\prime \prime} \in \mathcal{A}^{* *}$. For more details about Arens product, We refer to [4].

Throughout of this paper, for each $n \geq 0$, we consider $(2 n)$-th dual of Banach algebra $\mathcal{A}, \mathcal{A}^{(2 n)}$, with the first Arens product.

By Goldstine's theorem [4, p.425], there are nets $\left(a_{\alpha}\right)_{\alpha}$ and $\left(b_{\beta}\right)_{\beta}$ in $\mathcal{A}$ such that $a^{\prime \prime}=w e a k^{*}-\lim _{\alpha} a_{\alpha}$ and $b^{\prime \prime}=w e a k^{*}-\lim _{\beta} b_{\beta}$. So, it is easy to see that,

$$
a^{\prime \prime} \square b^{\prime \prime}=w^{*}-\lim _{\alpha} w^{*}-\lim _{\beta}\left(a_{\alpha} b_{\beta}\right)
$$

and

$$
a^{\prime \prime} \diamond b^{\prime \prime}=w^{*}-\lim _{\beta} w^{*}-\lim _{\alpha}\left(a_{\alpha} b_{\beta}\right) .
$$

Thus, $\mathcal{A}$ is Arens regular if and only if we have 


$$
w^{*}-\lim _{\alpha} w^{*}-\lim _{\beta}\left(a_{\alpha} b_{\beta}\right)=w^{*}-\lim _{\beta} w^{*}-\lim _{\alpha}\left(a_{\alpha} b_{\beta}\right)
$$

Also, we can find the first and second topological centers of $\mathcal{A}^{* *}$, which are

$$
\begin{aligned}
& Z_{\mathcal{A}}\left(\mathcal{A}^{* *}\right)=\left\{a^{\prime \prime} \in \mathcal{A}^{* *}: b^{\prime \prime} \mapsto a^{\prime \prime} \square b^{\prime \prime} \text { is weak }{ }^{*}-\text { weak } k^{*} \text { continuous }\right\} \\
& Z_{\mathcal{A}}^{t}\left(\mathcal{A}^{* *}\right)=\left\{b^{\prime \prime} \in \mathcal{A}^{* *}: a^{\prime \prime} \mapsto a^{\prime \prime} \diamond b^{\prime \prime} \text { is weak }{ }^{*}-\text { weak } k^{*} \text { continuous }\right\} .
\end{aligned}
$$

A Banach algebra $\mathcal{A}$ is Arens regular when $Z_{\mathcal{A}}\left(\mathcal{A}^{* *}\right)=\mathcal{A}^{* *}$, or equivalently, $Z_{\mathcal{A}}^{t}\left(\mathcal{A}^{* *}\right)=\mathcal{A}^{* *}$. (see, [6]).

Let $\mathcal{A}$ be a Banach algebra and $X$ is a Banach $\mathcal{A}$-bimodule. A derivation $D: \mathcal{A} \longrightarrow X$ is a continuous linear map such that for all $a, b \in \mathcal{A}$,

$$
D(a b)=a \cdot D(b)+D(a) \cdot b .
$$

For each $x \in X$, we define a map $D_{x}: \mathcal{A} \longrightarrow X$ by

$$
D_{x}(a)=a \cdot x-x \cdot a \quad(a \in \mathcal{A}) .
$$

It is easily seen that $D_{x}$ is a derivation. Such derivations are called inner derivations. $\mathcal{Z}^{1}(\mathcal{A}, X)$ is the Banach space of all continuous derivations from $\mathcal{A}$ into $X, \mathcal{B}^{1}(\mathcal{A}, X)$ is the normed space of all inner derivations from $\mathcal{A}$ into $X$. Then the first Hochschild cohomology group of $\mathcal{A}$ with coefficients in $X$ is the quotient space

$$
\mathcal{H}^{1}(\mathcal{A}, X)=\frac{\mathcal{Z}^{1}(\mathcal{A}, X)}{\mathcal{B}^{1}(\mathcal{A}, X)}
$$

With the above notations, a Banach algebra $\mathcal{A}$ is called amenable if $\mathcal{H}^{1}\left(\mathcal{A}, X^{*}\right)=0$ for every Banach $\mathcal{A}$-bimodule $X$. In fact, $\mathcal{A}$ is amenable if every derivation from $\mathcal{A}$ into $X^{*}$, is inner for every Banach $\mathcal{A}$-bimodule $X$. Also, Banach algebra $\mathcal{A}$ is weakly amenable ( $n$-weakly amenable) if $\mathcal{H}^{1}\left(\mathcal{A}, \mathcal{A}^{*}\right)=\{0\}\left(\mathcal{H}^{1}\left(\mathcal{A}, \mathcal{A}^{(n)}\right)=\{0\}\right)$, see $[4,5]$ for more details.

A Banach algebra $\mathcal{A}$ is dual if there is a closed submodule $\mathcal{A}_{*}$ of $\mathcal{A}^{*}$ such that $\mathcal{A}=\left(\mathcal{A}_{*}\right)^{*}$. Note that if $\mathcal{A}$ be a dual Banach algebra, then it is not necessary that the predual $\mathcal{A}_{*}$ is unique [8].

For example, if $\mathcal{A}$ be an Arens regular Banach algebra, then $\mathcal{A}^{* *}$ (with the first Arens product) is a dual Banach algebra with predual $\mathcal{A}^{*}$; if $G$ be a locally compact topological group then measure algebra $M(G)$ is a dual Banach algebra with predual $C_{0}(G)$; or if $E$ be a reflexive Banach algebra then $\mathcal{L}(E)$ is a dual Banach algebra with predual $E \widehat{\otimes} E^{*}$, where $\widehat{\otimes}$ is projective tensor product.

Now, let $\mathcal{A}$ be a dual Banach algebra and let $X^{*}$ be a dual Banach $\mathcal{A}$-bimodule. Then $f \in X^{*}$ is normal whenever for all $a \in \mathcal{A}$, mappings $a \mapsto a \cdot f$ and $a \mapsto f \cdot a$ be weak $k^{*}-w e a k^{*}-$ continuous from $\mathcal{A}$ into $X^{*}$. Also, $X^{*}$ is called normal if every element $f \in X^{*}$ is normal [8]. 
The dual Banach algebra $\mathcal{A}$ is called Connes amenable if every weak ${ }^{*}-$ weak $k^{*}$ continuous derivation from $\mathcal{A}$ into each normal dual Banach $\mathcal{A}$-bimodule $X^{*}$ is inner; i.e. $\mathcal{H}_{w^{*}}^{1}\left(\mathcal{A}, X^{*}\right)=\{0\}$. This concept was introduced by $\mathrm{V}$. Runde, [8].

Definition 1.1. A dual Banach algebra $\mathcal{A}$ is called weakly Connes amenable if every weak $k^{*}$ wea $k^{*}$ - continuous derivation from $\mathcal{A}$ into normal dual Banach $\mathcal{A}$-bimodule $\mathcal{A}^{*}$ is inner; that is $\mathcal{H}_{w^{*}}^{1}\left(\mathcal{A}, \mathcal{A}^{*}\right)=\{0\}$.

In this paper, We will study the weak Connes Amenability of the even duals of an Arens regular Banach algebra $\mathcal{A}$. We will do this work by induction.

\section{Weak Connes Amenability}

Let $\mathcal{A}$ be an Arens regular Banach algebra and $\mathcal{A}^{(2 n)}$ be its $(2 n)$-th dual for all $n \geq 0$. It is clear that $\mathcal{A}^{0}=\mathcal{A}$. In this section, we will show that how weak amenability of $\mathcal{A}^{(2 n)}$ and weak Connes amenability of $\mathcal{A}^{(2 n+2)}$ are related together. First, we present a general law about normality of modular structures.

Lemma 2.1. Let $\mathcal{A}$ be an Arens regular Banach algebra. Then $\mathcal{A}^{*}$ is a normal Banach $\mathcal{A}$-bimodule.

Proof. Let $\left(a_{\alpha}\right)_{\alpha}$ is a net in $\mathcal{A}$ and $a^{\prime} \in \mathcal{A}^{*}$. Then, by the Arens regularity of $\mathcal{A}$, we have

$$
\begin{aligned}
\left\langle\left(w^{*}-\lim _{\alpha} a_{\alpha}\right) \cdot a^{\prime}, b\right\rangle & =\left\langle a^{\prime}, b \cdot\left(w^{*}-\lim _{\alpha} a_{\alpha}\right)\right\rangle \\
& =\lim _{\alpha}\left\langle a^{\prime}, b \cdot a_{\alpha}\right\rangle \\
& =\lim _{\alpha}\left\langle a_{\alpha} \cdot a^{\prime}, b\right\rangle \\
& =\left\langle w^{*}-\lim _{\alpha}\left(a_{\alpha} \cdot a^{\prime}\right), b\right\rangle,
\end{aligned}
$$

for every $b \in \mathcal{A}$. Also,

$$
\begin{aligned}
\left\langle a^{\prime} \cdot\left(w^{*}-\lim _{\alpha} a_{\alpha}\right), b\right\rangle & =\left\langle a^{\prime}, w^{*}-\lim _{\alpha} a_{\alpha} \cdot b\right\rangle \\
& =\lim _{\alpha}\left\langle a^{\prime}, a_{\alpha} \cdot b\right\rangle \\
& =\lim _{\alpha}\left\langle a^{\prime} \cdot a_{\alpha}, b\right\rangle \\
& =\left\langle w^{*}-\lim _{\alpha}\left(a^{\prime} \cdot a_{\alpha}\right), b\right\rangle .
\end{aligned}
$$

Hence, mappings $a \mapsto a \cdot a^{\prime}$ and $a \mapsto a^{\prime} \cdot a$ are $w e a k^{*}-w e a k^{*}-$ continuous from $\mathcal{A}$ into $\mathcal{A}^{*}$. Consequently, $\mathcal{A}^{*}$ is a normal Banach $\mathcal{A}$-bimodule. 
Similarly, we have the following extended result.

Corollary 2.2. Let $\mathcal{A}$ be a Banach algebra and $n \geq 0$. If $\mathcal{A}^{(2 n)}$ is an Arens regular, then $\mathcal{A}^{(2 n+1)}$ is a normal Banach $\mathcal{A}^{(2 n)}$-bimodule.

Now, we prove the first theorem of this paper.

Theorem 2.3. Let $\mathcal{A}$ be an Arens regular Banach algebra such that every map from $\mathcal{A}$ into $\mathcal{A}^{*}$ is weakly compact. If $\mathcal{A}^{* *}$ is weakly Connes amenable, then $\mathcal{A}$ is a weakly Connes amenable.

Proof. Let $D: \mathcal{A} \rightarrow \mathcal{A}^{*}$ is weak $k^{*}-$ weak ${ }^{*}$-continuous derivation which $\mathcal{A}^{*}$ is a normal Banach $\mathcal{A}$-bimodule, by lemma 2.1 . It is easily seen that $D^{* *}: \mathcal{A}^{* *} \rightarrow \mathcal{A}^{(3)}$ is weak $k^{*}-w e a k^{*}$-continuous derivation by [4, theorem 2.8.59]. Since $\mathcal{A}^{* *}$ is weakly Connes amenable, so $D^{* *}$ is inner. Then there is $a^{(3)} \in \mathcal{A}^{(3)}$ such that for all $a^{\prime \prime} \in \mathcal{A}^{* *}$

$$
D^{* *}\left(a^{\prime \prime}\right)=a^{\prime \prime} \cdot a^{(3)}-a^{(3)} \cdot a^{\prime \prime} .
$$

Now, suppose that $K: \mathcal{A} \rightarrow \mathcal{A}^{* *}$ be a canonical map. Let $a^{\prime}=K^{*}\left(a^{(3)}\right)$. Then for each $a \in \mathcal{A}$

$$
D(a)=a \cdot a^{\prime}-a^{\prime} \cdot a .
$$

Therefore $D$ is inner and $\mathcal{A}$ is weakly Connes amenable.

Theorem 2.4. Let $\mathcal{A}$ be a Banach algebra such that every map from $\mathcal{A}^{* *}$ into $\mathcal{A}^{(3)}$ is weakly compact. If $\mathcal{A}^{* *}$ is an Arens regular and $\mathcal{A}^{(4)}$ is weakly Connes amenable, then $\mathcal{A}^{* *}$ is a weakly Connes amenable.

Proof. Let $D: \mathcal{A}^{* *} \rightarrow \mathcal{A}^{(3)}$ is weak $k^{*}-w e a k^{*}$-continuous derivation which $\mathcal{A}^{(3)}$ is a normal Banach $\mathcal{A}^{* *}$-bimodule, by corollary 2.2 . It is easily seen that $D^{* *}: \mathcal{A}^{(4)} \rightarrow \mathcal{A}^{(5)}$ is weak $k^{*}-w e a k^{*}$-continuous derivation [4]. Since $\mathcal{A}^{(4)}$ is weakly Connes amenable, so $D^{* *}$ is inner. Then there is $a^{(5)} \in \mathcal{A}^{(5)}$ such that for all $a^{(4)} \in \mathcal{A}^{(4)}$

$$
D^{* *}\left(a^{(4)}\right)=a^{(4)} \cdot a^{(5)}-a^{(5)} \cdot a^{(4)} .
$$

Now, suppose that $K: \mathcal{A}^{* *} \rightarrow \mathcal{A}^{(4)}$ be a canonical map. Let $a^{(3)}=K^{*}\left(a^{(5)}\right)$. Then for each $a^{\prime \prime} \in \mathcal{A}^{* *}$

$$
D\left(a^{\prime \prime}\right)=a^{\prime \prime} \cdot a^{(3)}-a^{(3)} \cdot a^{\prime \prime}
$$

Therefore $D$ is inner and $\mathcal{A}^{* *}$ is weakly Connes amenable.

Now, by the two theorems 2.3 and 2.4, we can state the next theorem inductively. 
Theorem 2.5. Let $\mathcal{A}$ be a Banach algebra and $n \geq 0$. Also, let every map from $\mathcal{A}^{(2 n)}$ into $\mathcal{A}^{(2 n+1)}$ is weakly compact. If $\mathcal{A}^{(2 n)}$ is an Arens regular and $\mathcal{A}^{(2 n+2)}$ is weakly Connes amenable, then $\mathcal{A}^{(2 n)}$ is a weakly Connes amenable.

Proof. Proof is similar to proofs of two last theorems.

In the next, we can see that weak amenability of an arbitrary Banach algebra implies weak Connes amenability of its even duals.

Theorem 2.6. Let $\mathcal{A}$ be a Banach algebra and $\mathcal{A}^{* *}$ is an Arens regular. If $\mathcal{A}$ is weakly amenable, then $\mathcal{A}^{* *}$ is weakly Connes amenable.

Proof. Let $D: \mathcal{A}^{* *} \rightarrow \mathcal{A}^{(3)}$ be a weak - weak $k^{*}$-continuous derivation which by corollary $2.2, \mathcal{A}^{(3)}$ is a normal Banach $\mathcal{A}^{* *}$-bimodule. For each $a \in \mathcal{A}$, we define $\tilde{D}: \mathcal{A} \rightarrow \mathcal{A}^{*}$ by

$$
\tilde{D}(a)=\left.D(\hat{a})\right|_{\mathcal{A}}
$$

For every $a, b \in \mathcal{A}$, we have

$$
\tilde{D}(a b)=D(\widehat{a b})=D(\hat{a} \square \hat{b})=a D(\hat{b})+D(\hat{a}) b=a \tilde{D}(b)+\tilde{D}(a) b .
$$

Thus $\tilde{D}$ is a continuous derivation from $\mathcal{A}$ into $\mathcal{A}^{*}$. By hypothesis, since $\mathcal{A}$ is weakly amenable, thus $\tilde{D}$ is inner. Then

$$
D(\hat{a})=\tilde{D}(a)=\left.a \cdot a^{(3)}\right|_{\mathcal{A}}-\left.a^{(3)}\right|_{\mathcal{A}} \cdot a=\left.\hat{a} \cdot a^{(3)}\right|_{\mathcal{A}}-\left.a^{(3)}\right|_{\mathcal{A}} \cdot \hat{a} .
$$

Considering natural mapping $K: \mathcal{A}^{*} \rightarrow \mathcal{A}^{(3)}$, there is $b^{(3)} \in \mathcal{A}^{(3)}$ such that $K\left(\left.a^{(3)}\right|_{\mathcal{A}}\right)=b^{(3)}$. So

$$
D(\hat{a})=\hat{a} \cdot b^{(3)}-b^{(3)} \cdot \hat{a} .
$$

Therefore $D$ is inner. Consequently, $\mathcal{A}^{* *}$ is weakly Connes amenable.

Theorem 2.7. Let $\mathcal{A}$ be a Banach algebra. Also, suppose that $\mathcal{A}^{(4)}$ is an Arens regular. If $\mathcal{A}^{* *}$ is weakly amenable, then $\mathcal{A}^{(4)}$ is weakly Connes amenable.

Proof. Let $D: \mathcal{A}^{(4)} \rightarrow \mathcal{A}^{(5)}$ be a weak ${ }^{*}-w e a k^{*}$-continuous derivation which by corollary $2.2, \mathcal{A}^{(5)}$ is a normal Banach $\mathcal{A}^{(4)}$-bimodule. For each $a^{\prime \prime} \in \mathcal{A}^{* *}$, we define $\tilde{D}: \mathcal{A}^{* *} \rightarrow \mathcal{A}^{(3)}$ by follow

$$
\tilde{D}\left(a^{\prime \prime}\right)=\left.D\left(\widehat{a^{\prime \prime}}\right)\right|_{\mathcal{A}^{* *}}
$$

For every $a^{\prime \prime}, b^{\prime \prime} \in \mathcal{A}^{* *}$, we have

$$
\tilde{D}\left(a^{\prime \prime} \square b^{\prime \prime}\right)=D\left(\widehat{a^{\prime \prime} b^{\prime \prime}}\right)=D\left(\widehat{a^{\prime \prime}} \square \widehat{b^{\prime \prime}}\right)=a^{\prime \prime} D\left(\widehat{b^{\prime \prime}}\right)+D\left(\widehat{a^{\prime \prime}}\right) b^{\prime \prime}=a^{\prime \prime} \tilde{D}\left(b^{\prime \prime}\right)+\tilde{D}\left(a^{\prime \prime}\right) b^{\prime \prime} .
$$


Thus $\tilde{D}$ is a continuous derivation from $\mathcal{A}^{* *}$ into $\mathcal{A}^{(3)}$. By hypothesis, since $\mathcal{A}^{* *}$ is weakly amenable, thus $\tilde{D}$ is inner. Then

$$
D\left(\widehat{a^{\prime \prime}}\right)=\tilde{D}\left(a^{\prime \prime}\right)=\left.a^{\prime \prime} \cdot a^{(5)}\right|_{\mathcal{A}^{* *}}-\left.a^{(5)}\right|_{\mathcal{A}^{* *}} \cdot a^{\prime \prime}=\left.\widehat{a^{\prime \prime}} \cdot a^{(5)}\right|_{\mathcal{A}^{* *}}-\left.a^{(5)}\right|_{\mathcal{A}^{* *}} \cdot \widehat{a^{\prime \prime}}
$$

Considering natural mapping $K: \mathcal{A}^{(3)} \rightarrow \mathcal{A}^{(5)}$, there is $b^{(5)} \in \mathcal{A}^{(5)}$ such that $K\left(\left.a^{(5)}\right|_{\mathcal{A}^{* *}}\right)=b^{(5)}$. So

$$
D\left(\widehat{a^{\prime \prime}}\right)=\widehat{a^{\prime \prime}} \cdot b^{(5)}-b^{(5)} \cdot \widehat{a^{\prime \prime}}
$$

Therefore $D$ is inner. Consequently, $\mathcal{A}^{(4)}$ is weakly Connes amenable.

According to the above theorems, we have the following general theorem. So, we omit the proof.

Theorem 2.8. Let $\mathcal{A}$ be a Banach algebra. Also, suppose that $\mathcal{A}^{(2 n+2)}$ is an Arens regular. If $\mathcal{A}^{(2 n)}$ is weakly amenable, then $\mathcal{A}^{(2 n+2)}$ is weakly Connes amenable.

Let $\mathcal{A}$ be a Banach algebra and $\mathcal{A}^{*}$ is a Banach $\mathcal{A}$-bimodule. We say that $\mathcal{A}^{*}$ factors on the left (right) if $\mathcal{A}^{*}=\mathcal{A}^{*} \cdot \mathcal{A}\left(\mathcal{A}^{*}=\mathcal{A} \cdot \mathcal{A}^{*}\right)$ and $\mathcal{A}^{*}$ factors if both equalities $\mathcal{A} \cdot \mathcal{A}^{*}=\mathcal{A}^{*}=\mathcal{A}^{*} \cdot \mathcal{A}$ hold $[1]$.

In general, If $\mathcal{A}^{(2 n+1)}$ be a Banach $\mathcal{A}^{(2 n)}$-bimodule, then we say that $\mathcal{A}^{(2 n+1)}$ factors if $\mathcal{A}^{(2 n)} \cdot \mathcal{A}^{(2 n+1)}=\mathcal{A}^{(2 n+1)}=\mathcal{A}^{(2 n+1)} \cdot \mathcal{A}^{(2 n)}$. Now, by applying this property, we show that even duals of $\mathcal{A}$ is weakly Connes amenable, under some conditions.

Theorem 2.9. Let $\mathcal{A}$ be weakly amenable Banach algebra. If $\mathcal{A}^{* *}$ is the left ideal in $\mathcal{A}^{(4)}$ and $\mathcal{A}^{(3)}$ factors on the right, then $\mathcal{A}^{* *}$ is weakly Connes amenable.

Proof. Consider $\mathcal{A}^{(3)}$ as a Banach $\mathcal{A}^{* *}$-bimodule. Let $b^{(4)} \in \mathcal{A}^{(4)}$. Also, let $\left(a_{\alpha}^{\prime \prime}\right)_{\alpha}$ be a net in $\mathcal{A}^{* *}$ such that $\widehat{a_{\alpha}^{\prime \prime}} \stackrel{w^{*}}{\longrightarrow} \widehat{a^{\prime \prime}}$ in $\mathcal{A}^{(4)}$. We show that $b^{(4)} \widehat{a_{\alpha}^{\prime \prime}} \stackrel{w^{*}}{\longrightarrow}$ $b^{(4)} a^{\prime \prime}$ in $\mathcal{A}^{(4)}$. Let $a^{(3)} \in \mathcal{A}^{(3)}$. Then, since $\mathcal{A}^{(3)}$ factors on the right, there are $b^{\prime \prime} \in \mathcal{A}^{* *}$ and $b^{(3)} \in \mathcal{A}^{(3)}$ such that $a^{(3)}=b^{\prime \prime} b^{(3)}$. Since $\mathcal{A}^{* *}$ is the left ideal in $\mathcal{A}^{(4)}$, thus we have $\widehat{a_{\alpha}^{\prime \prime}} b^{\prime \prime} \stackrel{w^{*}}{\longrightarrow} \widehat{a^{\prime \prime}} b^{\prime \prime}$ in $\mathcal{A}^{(4)}$ if and only if $\widehat{a_{\alpha}^{\prime \prime}} b^{\prime \prime} \stackrel{w^{*}}{\longrightarrow} \widehat{a^{\prime \prime}} b^{\prime \prime}$ in $\mathcal{A}^{* *}$. Also. Since $b^{\prime \prime} \mapsto b^{\prime \prime} b^{(3)} ; \mathcal{A}^{* *} \rightarrow \mathcal{A}^{(3)}$ is weak $k^{*}-w e a k^{*}$-continuous mapping, it is follows that $\widehat{a_{\alpha}^{\prime \prime}} b^{\prime \prime} b^{(3)} \stackrel{w^{*}}{\longrightarrow} \widehat{a^{\prime \prime}} b^{\prime \prime} b^{(3)}$ in $\mathcal{A}^{(3)}$. Therefore

$$
\begin{aligned}
\lim _{\alpha}\left\langle b^{(4)} \widehat{a_{\alpha}^{\prime \prime}}, a^{(3)}\right\rangle & =\lim _{\alpha}\left\langle b^{(4)} \widehat{a_{\alpha}^{\prime \prime}}, b^{\prime \prime} b^{(3)}\right\rangle \\
& =\lim _{\alpha}\left\langle b^{(4)}, \widehat{a_{\alpha}^{\prime \prime}} b^{\prime \prime} b^{(3)}\right\rangle \\
& =\left\langle b^{(4)}, \widehat{a^{\prime \prime}} b^{\prime \prime} b^{(3)}\right\rangle \\
& =\left\langle b^{(4)} \widehat{a^{\prime \prime}}, b^{\prime \prime} b^{(3)}\right\rangle \\
& =\left\langle b^{(4)} \widehat{a^{\prime \prime}}, a^{(3)}\right\rangle .
\end{aligned}
$$


This shows that $b^{(4)} \in Z_{\mathcal{A}^{* *}}\left(\mathcal{A}^{(4)}\right)$. Hence $\mathcal{A}^{* *}$ is Arens regular. By the hypothesis, since $\mathcal{A}$ is weakly amenable, so imediately theorem 2.6 implies that $\mathcal{A}^{* *}$ is weakly Connes amenable.

Theorem 2.10. Let $\mathcal{A}$ be weakly amenable Banach algebra. If $\mathcal{A}^{* *}$ is the right ideal in $\mathcal{A}^{(4)}$ and $\mathcal{A}^{(3)}$ factors on the left, then $\mathcal{A}^{* *}$ is weakly Connes amenable.

Proof. Consider $\mathcal{A}^{(3)}$ as a Banach $\mathcal{A}^{* *}$-bimodule. Let $b^{(4)} \in \mathcal{A}^{(4)}$. Also, let $\left(a_{\alpha}^{\prime \prime}\right)_{\alpha}$ be a net in $\mathcal{A}^{* *}$ such that $\widehat{a_{\alpha}^{\prime \prime}} \stackrel{w^{*}}{\longrightarrow} \widehat{a^{\prime \prime}}$ in $\mathcal{A}^{(4)}$. We show that $b^{(4)} \widehat{a_{\alpha}^{\prime \prime}} \stackrel{w^{*}}{\longrightarrow}$ $b^{(4)} a^{\prime \prime}$ in $\mathcal{A}^{(4)}$. Let $a^{(3)} \in \mathcal{A}^{(3)}$. Then, since $\mathcal{A}^{(3)}$ factors on the left, there are $b^{\prime \prime} \in \mathcal{A}^{* *}$ and $b^{(3)} \in \mathcal{A}^{(3)}$ such that $a^{(3)}=b^{(3)} b^{\prime \prime}$. Since $\mathcal{A}^{* *}$ is the right ideal in $\mathcal{A}^{(4)}$, thus we have $b^{\prime \prime} \widehat{a_{\alpha}^{\prime \prime}} \stackrel{w^{*}}{\longrightarrow} b^{\prime \prime} \widehat{a}^{\prime \prime}$ in $\mathcal{A}^{(4)}$ if and only if $b^{\prime \prime} \widehat{a_{\alpha}^{\prime \prime}} \stackrel{w^{*}}{\longrightarrow} b^{\prime \prime} \widehat{a}^{\prime \prime}$ in $\mathcal{A}^{* *}$. Also. Since $b^{\prime \prime} \mapsto b^{(3)} b^{\prime \prime} ; \mathcal{A}^{* *} \rightarrow \mathcal{A}^{(3)}$ is weak $k^{*}-w e a k^{*}$-continuous mapping, it is follows that $\widehat{a_{\alpha}^{\prime \prime}} b^{(3)} b^{\prime \prime} \stackrel{w^{*}}{\longrightarrow} \widehat{a^{\prime \prime}} b^{(3)} b^{\prime \prime}$ in $\mathcal{A}^{(3)}$. Therefore

$$
\begin{aligned}
\lim _{\alpha}\left\langle b^{(4)} \widehat{a_{\alpha}^{\prime \prime}}, a^{(3)}\right\rangle & =\lim _{\alpha}\left\langle b^{(4)} \widehat{a_{\alpha}^{\prime \prime}}, b^{(3)} b^{\prime \prime}\right\rangle \\
& =\lim _{\alpha}\left\langle b^{(4)}, \widehat{a_{\alpha}^{\prime \prime}} b^{(3)} b^{\prime \prime}\right\rangle \\
& =\left\langle b^{(4)}, \widehat{a^{\prime \prime}} b^{(3)} b^{\prime \prime}\right\rangle \\
& =\left\langle b^{(4)} \widehat{a^{\prime \prime}}, b^{(3)} b^{\prime \prime}\right\rangle \\
& =\left\langle b^{(4)} \widehat{a^{\prime \prime}}, a^{(3)}\right\rangle .
\end{aligned}
$$

This shows that $b^{(4)} \in Z_{\mathcal{A}^{* *}}\left(\mathcal{A}^{(4)}\right)$. Hence $\mathcal{A}^{* *}$ is Arens regular. By the hypothesis, since $\mathcal{A}$ is weakly amenable, so imediately theorem 2.6 implies that $\mathcal{A}^{* *}$ is weakly Connes amenable.

Corollary 2.11. Let $\mathcal{A}$ is weakly amenable Banach algebra. If $\mathcal{A}^{* *}$ is a two-sided ideal in $\mathcal{A}^{(4)}$ and $\mathcal{A}^{(3)}$ factors, then $\mathcal{A}^{* *}$ is weakly Connes amenable. Proof. It is easily followed from Theorems 2.9 and 2.10 .

Note that condition of factorization of Banach $\mathcal{A}^{* *}$-bimodule $\mathcal{A}^{(3)}$ is necessary in above results. [7]

Finally, by according to corollary 2.11 we can bring the following theorem in general. Of course, note that $n$ is a non-negative number.

Theorem 2.12. Let $\mathcal{A}^{(2 n)}$ be weakly amenable Banach algebra. If $\mathcal{A}^{(n+2)}$ is a two-sided ideal in $\mathcal{A}^{(n+4)}$ and $\mathcal{A}^{(n+3)}$ factors, then $\mathcal{A}^{(2 n+2)}$ is weakly Connes amenable.

Proof. It is easily followed from the above theorems. 


\section{References}

[1] A. T. M. Lau and A. Ulger, Topological center of certain dual algebras, Trans. Amer. Math. Soc, 348(1996), 1191 - 1212.

[2] A. Ya. Helemskii, A certain class of flat Banach modules and its applications, vest. Mosk Univ. ser. Mat. Mekh, 27(1972), 29 - 36.

[3] B. E. Johnson, Cohomology in Banach algebras, Mem. Amer. Math. Soc, 127(1972).

[4] H. G. Dales, Banach algebras and automatic continuity, London Math. Soc, Monographs, 24, Clarenden Press, Oxford, (2000).

[5] H. G. Dales, F. Ghahramani and N. Gronbaek, Derivations into iterated duals of Banach algebras, Studia Math, 128(1998), no.1, 19 - 54.

[6] M. Eshaghi Gordji and M. Filali, Arens regularity of module actions, Studia Math, 181(2007), 237 - 254.

[7] N. J. Young, The irregularity of multiplication in group algebras, Quart, J. Math. Oxford, 24(1973), 59 - 62.

[8] V. Runde, Amenability for dual Banach algebras, Studia Math, 148(2001), 47 - 66.

Received: March 16, 2013 